\title{
Caracterização dos danos causados por Heilipodus naevulus em plantios de eucalipto no Espírito Santo, Brasil
}

\author{
Characterization of damages caused by Heilipodus naevulus in eucalypt plantations on Espírito Santo, \\ Brazil
}

\author{
Reginaldo Gonçalves Mafia $^{I^{*}}$ João Bosco da Silva ${ }^{I}$ Jurandir Floriano Ramos
}

- NOTA -

\section{RESUMO}

O objetivo do presente trabalho foi avaliar os efeitos dos danos causados por Heilipodus naevulus Mann. (Coleoptera: Curculionidae) no desenvolvimento de plantios jovens de eucalipto, além de relatar, pela primeira vez, a ocorrência desse inseto no estado do Espírito Santo. O período crítico para ocorrência foi caracterizado entre outubro a dezembro, de acordo com o regime de chuvas. Os danos causados pela praga reduziram o desenvolvimento e a qualidade das árvores de eucalipto.

Palavras-chave: entomologia florestal, gorgulho, curculionídeos.

\begin{abstract}
This research aimed to evaluate the effects of damages caused by Heilipodus naevulus Mann. (Coleoptera: Curculionidae) to the development of eucalyptus young plantation, and to record, for the first time, the occurrence on Espírito Santo State, Brazil. The critical period of occurrence was observed between October to December according with rain regime. The damages caused by the pest reduced the development and quality of eucalyptus trees.
\end{abstract}

Key words: forest entomology, weevils, curculionids.

Os besouros desfolhadores são importantes pragas da cultura do eucalipto. No Brasil, os registros de surtos de curculionídeos têm se tornado mais frequentes, incluindo as espécies Heilipodus naevulus (Mannerheim, 1836) (MATRANGOLO et al., 2008), Heilipodus dorsosulcatus (Boheman, 1843) (GARLET et al., 2011) e Chalcodermus bicolor (Fiedler, 1936)
(SOUZA et al., 2011). Sendo assim, o objetivo do presente trabalho foi avaliar os efeitos dos danos causados por $\boldsymbol{H}$. naevulus no desenvolvimento de plantios jovens de eucalipto no Estado do Espírito Santo, além de relatar, pela primeira vez, a ocorrência desse inseto no Estado.

O primeiro registro de $\boldsymbol{H}$. naevulus em eucalipto, provavelmente, foi realizado em 1974 por Morais e Berti Filho (MORAES \& BERTI FILHO, 1974), quando esses autores realizaram uma ampla coleta $\mathrm{e}$ identificação de coleobrocas que ocorrem em essências florestais. Naquela época, os autores relataram danos causados por adultos de Heilipus sp. em Eucalyptus saligna, no Estado de São Paulo. Considerando a reclassificação e transferência de algumas espécies de Heilipus para o gênero Heilipodus (KUSCHEL, 1955), acredita-se que esse tenha sido provavelmente o primeiro registro de danos causados por H. naevulus. No Espírito Santo, outra espécie de Curculionidae também foi relatada na mesma época, em Eucalyptus robusta e $\boldsymbol{E}$. saligna. Todavia, de acordo com a descrição do padrão de danos e comportamento dos insetos, acredita-se que a espécie relatada naquela oportunidade deveria ser o que mais tarde foi identificado como Chalcodermus bicolor (SOUZA et al., 2011).

O besouro $\boldsymbol{H}$. naevulus, denominado de bicudo, gorgulho dos ponteiros, maromba ou trombeta, é uma praga ocasional para o eucalipto. Os insetos adultos têm, aproximadamente, $12 \mathrm{~mm}$ de comprimento,

IFibria S.A. Centro de Tecnologia, Rod. Aracruz Barra do Riacho, Km 25, 29197-900. Aracruz, ES, Brasil. E-mail: rgoncalves@ fibria.com.br. *Autor para correspondência. 
cabeça projetada para frente, cor castanho-escura, apresentando uma mancha preta na segunda metade de cada élitro e, logo abaixo, uma saliência com pubescência de cor branca, no caso de $\boldsymbol{H}$. naevulus, e de cor quase preta uniforme, no caso de $\boldsymbol{H}$. dorsosulcatus. Os adultos apresentam vôos curtos e possuem o hábito noturno. Durante o dia, os insetos permanecem escondidos em detritos no solo ou na base dos ramos de eucalipto. Além do eucalipto, H. naevulus é um importante inseto-praga da videira (Vitis vinifera) e causa danos também em cajueiro (Anacardium occidentale), espirradeira (Nerium oleander), marmeleiro (Pyrus cydonia) e nespeira (Eriobotrya japonica) (SILVA et al., 1968). Ainda não se conhece em detalhes o ciclo evolutivo de $\boldsymbol{H}$. naevulus. As posturas são realizadas no solo, sendo que o desenvolvimento das larvas ocorre nesse mesmo ambiente. Em condições de laboratório, o ciclo de vida foi de 14 meses (SORIA \& DAL CONTE, 2005).

A detecção da praga ocorreu em plantas de dois clones de eucalipto, híbridos Eucalyptus grandis $\mathrm{x}$
Eucalyptus urophylla, em um plantio experimental com quatro meses de idade, localizado em Aracruz, ES (1949'13" S, 40¹6'25" W e 60m de altitude). A primeira verificação ocorreu em outubro de 2011. O ataque do curculionídeo ocorreu na parte superior da copa, primeiramente na porção apical do ponteiro principal e, posteriormente, nas extremidades dos ramos laterais, no sentido ápice-base. $\mathrm{O}$ inseto causou perfurações no sentido transversal e raspagem do tecido vegetal em vários pontos. Os ramos danificados murcharam de cima para baixo, apodreceram e, na sequência, secaram, sendo que, em alguns casos, quebraram com o próprio peso ou pela ação do vento. Em função da morte do meristema apical, as plantas danificadas bifurcaram, em diferentes alturas, dependendo do porte da planta quando esta foi atacada. Várias plantas foram danificadas em mais de um momento e apresentaram bifurcações em mais de um ponto ao longo do fuste (Figura 1).

Aos nove meses de idade, os plantios dos dois clones atacados foram avaliados quanto ao desenvolvimento em altura total (HT), circunferência a

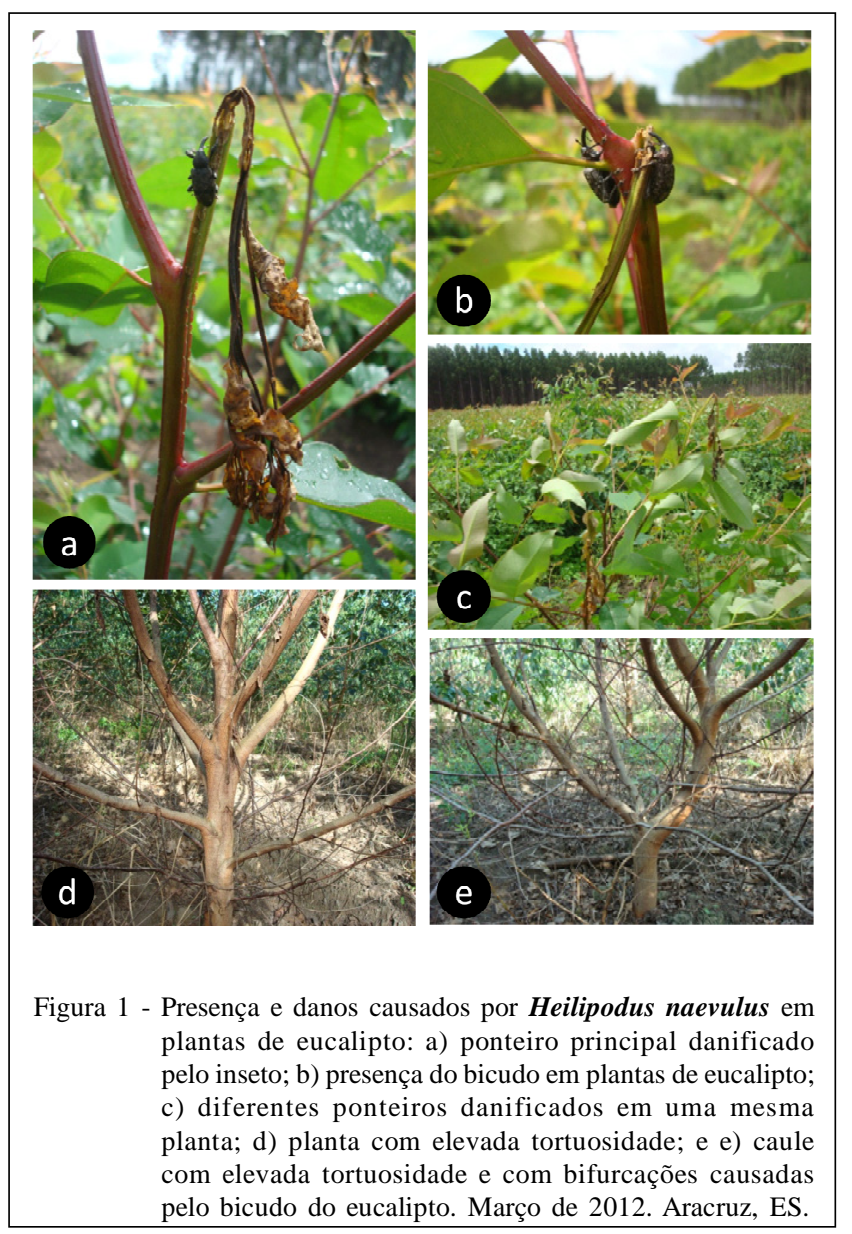

Ciência Rural, v.43, n.2, fev, 2013. 
altura do peito (CAP) e altura em que houve bifurcação (HB) do fuste. Para plantas bifurcadas, o CAP foi determinado para o fuste com maior desenvolvimento. Para plantas com bifurcação em mais de um ponto, considerou-se como HB a altura para o primeiro ponto de bifurcação, no sentido solo ápice da planta. Para medição, foram alocadas, ao acaso, seis parcelas de 33 plantas para cada clone de eucalipto. As plantas avaliadas foram segregadas em classes de crescimento, considerando a HT. Em cada classe de altura, foi determinado o percentual de plantas bifurcadas, a média de HB, o CAP médio geral (plantas bifurcadas e normais), o CAP das plantas bifurcadas e das árvores normais, ou seja, que não foram danificadas pelo inseto.

As características morfológicas dos insetos coletados foram contrastadas com a descrição da espécie e com exemplares identificados pelo Dr. Germano H. Rosado Neto (UNIVERSIDADE FEDERAL DO PARANÁ), coletados na mesma região em 1991, comprovando tratar-se de $\boldsymbol{H}$. naevulus. Adultos de ambos os sexos, diferenciados pelo formato do prémucro da tíbia mediana (MATRANGOLO, 2010) foram depositados na coleção entomológica do Centro de Tecnologia da empresa florestal Fibria, localizada em Aracruz, ES.

A detecção do inseto $\boldsymbol{H}$. naevulus, historicamente na região de estudo, tem ocorrido normalmente no início da estação chuvosa, ou seja, a partir de setembro a outubro, coincidindo com o período favorável para outros besouros desfolhadores, principalmente Costalimita ferruginea (Fabricius, 1801) (Coleoptera: Chrysomelidae) (SOUZA et al., 2008). A média de precipitação pluviométrica na região de estudo foi de $138 \mathrm{~mm}$ de chuvas no mês de outubro, considerando uma série histórica de dados de 40 anos. Para o mesmo mês em 2011, ocorreram aproximadamente 206mm de chuvas (dados não publicados; Fonte: Fibria Celulose S.A.). Desse modo, acredita-se que o período crítico para ocorrência de $\boldsymbol{H}$. naevulus ocorre de outubro a dezembro, podendo se estender até o mês de março do ano seguinte, dependendo do regime de chuvas.

A incidência e o nível de danos causados pelo bicudo do eucalipto foram maiores para um dos clones avaliados (FBR61) (Tabela 1). Todavia, considerando que os surtos dessa praga ocorrem de forma agregada (MATRANGOLO, 2010), acredita-se que não houve preferência do inseto em relação ao material genético e que as diferenças no nível populacional tenham sido mais relacionadas com o posicionamento geográfico dos plantios. O clone mais afetado foi plantado na extremidade do talhão experimental, vizinho de uma área de preservação de floresta nativa.

O crescimento inicial e a uniformidade dos plantios, aos nove meses de idade, foram afetados pelos danos causados pelo bicudo do eucalipto. A altura do clone FBR61 variou de 2 a $7 \mathrm{~m}$ de altura, enquanto o desenvolvimento do clone FBR84 foi mais uniforme, variando de 4 a $7 \mathrm{~m}$ (Tabela 1). $\mathrm{O}$ ataque da praga resultou em redução da altura de 24,8 e 11,9\%, respectivamente. Adicionalmente, observou-se que, nas maiores classes de crescimento, houve um menor percentual de plantas bifurcadas. Nas menores classes de crescimento do clone FBR61, entre 2 e $5 \mathrm{~m}$ de altura, $100 \%$ das plantas estavam bifurcadas, enquanto na maior classe, de 6 a $7 \mathrm{~m}$, o percentual foi de aproximadamente $85,71 \%$. Para o

Tabela 1 - Desenvolvimento inicial de plantios de eucalipto atacados por Heilipodus naevulus em função da classe de crescimento em altura. Maio de 2012. Aracruz, ES.

\begin{tabular}{ccccccc}
\hline Clone & Classe & $\begin{array}{c}\text { Bifurcadas } \\
(\%)\end{array}$ & $\begin{array}{c}\text { Altura da bifurcação } \\
(\mathrm{m}) *\end{array}$ & $\begin{array}{c}\text { CAP** Geral } \\
(\mathrm{cm})\end{array}$ & $\begin{array}{c}\text { CAP Bifurcadas } \\
(\mathrm{cm}) * * *\end{array}$ & $\begin{array}{c}\text { CAP Normal } \\
(\mathrm{cm}) * * *\end{array}$ \\
\hline \multirow{2}{*}{ FBR61 } & $2,0-3 \mathrm{~m}$ & 100,00 & $0,61 \mathrm{a}$ & 5,10 & 5,10 & - \\
& $3,1-4 \mathrm{~m}$ & 100,00 & $0,91 \mathrm{~b}$ & 8,60 & 8,60 & - \\
& $4,1-5 \mathrm{~m}$ & 100,00 & $1,14 \mathrm{~b}$ & 11,60 & 11,60 & - \\
& $5,1-6 \mathrm{~m}$ & 95,16 & $1,44 \mathrm{c}$ & 13,90 & $13,80 \mathrm{a}$ & $14,80 \mathrm{a}$ \\
& $6,1-7 \mathrm{~m}$ & 85,71 & $1,78 \mathrm{~d}$ & 15,80 & $15,50 \mathrm{a}$ & $17,80 \mathrm{~b}$ \\
& & & & & $11,30 \mathrm{a}$ & $12,40 \mathrm{~b}$ \\
FBR84 & $4,1-5 \mathrm{~m}$ & 76,19 & $0,81 \mathrm{a}$ & 11,60 & $14,00 \mathrm{a}$ & $15,40 \mathrm{~b}$ \\
& $5,1-6 \mathrm{~m}$ & 32,58 & $0,87 \mathrm{a}$ & 14,90 & $15,30 \mathrm{a}$ & $16,70 \mathrm{~b}$ \\
\hline
\end{tabular}

*Médias seguidas pela mesma letra para a variável altura de bifurcação e para um mesmo clone não diferem entre si pelo teste de Tukey (5\% de probabilidade de erro); **CAP: Circunferência do tronco, medido à altura do peito $(1,70 \mathrm{~m})$; ***Médias seguidas pela mesma letra para CAP entre plantas bifurcadas e normais, para um mesmo clone e classe de crescimento, não diferem entre si pelo teste $\mathrm{T}$ (5\% de probabilidade de erro). 
clone FBR84, na menor e maior classe, o percentual de plantas bifurcadas foi de 76,2 e $3,6 \%$, respectivamente. Ocorreu uma relação inversa para crescimento em altura e média da altura em que a bifurcação ocorreu, evidenciando que o dano mais precoce ou em plantas com menor desenvolvimento apresentam maior efeito adverso para o crescimento inicial do plantio. Para as variáveis de CAP, observou-se o mesmo padrão de resposta. A média de CAP das plantas normais, ou seja, não danificadas pela praga, em geral foi maior que a média geral (CAP Geral). O CAP das plantas bifurcadas foi igual ou menor que a média do CAP geral, comprovando o efeito da praga sobre o crescimento de ambos os clones. A maior diferença de crescimento em diâmetro ocorreu entre plantas bifurcadas e normais nas maiores classes de crescimento em altura. Em média, os danos causados pela praga reduziram em 12,9 e 8,4\% o crescimento em diâmetro para os clones FBR61 e FBR84 na maior classe de crescimento, respectivamente (Tabela 1), diferindo dos resultados obtidos por MATRANGOLO (2010), que não observou redução do crescimento inicial de plantios de eucalipto após o ataque de H. naevulus. Essa diferença pode estar relacionada com interações diferenciadas dos fatores apontados por COLLETT \& NEUMANN (2002), pela resposta fenotípica do material genético ou mesmo por diferenças na abordagem de avaliar os efeitos do ataque dessa praga. Quanto ao último aspecto, é importante mencionar que o ataque do bicudo do eucalipto pode ocorrer em plantas com diferentes portes, de uma única vez ou de forma sucessiva. A perda de dominância apical causa a bifurcação das plantas e, consequentemente, acarreta alterações morfológicas, incluindo normalmente o engrossamento do caule abaixo do ponto de bifurcação e alterações da relação hipsométrica.

Além de afetar o desenvolvimento inicial, as plantas atacadas, em função da perda de dominância apical, tiveram um comprometimento da forma do fuste, com aumento da tortuosidade. Acredita-se que esses pontos de bifurcação e tortuosidade devem afetar o crescimento das plantas, por maior restrição da passagem de água e nutrientes, podendo refletir negativamente sobre a produtividade ao final do ciclo. A presença de mais de um fuste por planta compromete o crescimento e o rendimento operacional da colheita mecanizada. Além disso, plantas bifurcadas são mais propensas à quebra pelo vento. Portanto, torna-se necessário realizar podas corretivas, o que onera o custo de formação florestal.

Os resultados obtidos no presente trabalho permitem concluir que o período crítico para os surtos de $\boldsymbol{H}$. naevulus no Estado do Espírito Santo ocorre entre os meses de outubro a dezembro e, dependendo do regime de chuvas, pode se estender até o mês de março do ano seguinte. Os danos causados pela praga resultam em bifurcação das plantas, aumento da tortuosidade do caule e redução do desenvolvimento inicial das plantas atacadas, o que pode comprometer a produtividade final do plantio, de acordo com o que foi observado neste primeiro relato da ocorrência dessa praga no Estado do Espírito Santo.

\section{REFERÊNCIAS}

COLLETT, N.G.; NEUMANN, F.G. Effects of simulated chronic defoliation in summer on growth and survival of blue gum (Eucalyptus globulus Labill.) within young plantations in northern Victoria. Australian Forestry, v.65, n.2, p.99-106, 2002. Disponível em: <http://www.forestry.org.au/ifa/c/c5ifa.asp>. Acesso em 11 abr. 2012.

GARLET, J. et al. First report of Heilipodus dorsosulcatus (Boheman, 1843) (Coleoptera: Curculionidae) in a plantation of eucalyptus L'Héritier in Brazil. Coleopterists Bulletin, v.65, n.3, p.243-245, 2011. Disponível em: <http://www.bioone.org/ doi/abs/10.1649/072.065.0306>. Acesso em 11 abr. 2012. doi: $10.1649 / 072.065 .0306$

KUSCHEL, G. Nuevas sinonimias y anotaciones sobre Curcuilionoidea (Coleoptera). Revista Chilena de Entomologia, v.4, p.261-312, 1955.

MATRANGOLO, C.A.R. et al. Ocorrência de Heilipodus naevulus Mannerheim (Curculionidae: Hylobiinae) em Minas Gerais. In: CONGRESSO BRASILEIRO DE ENTOMOLOGIA, 22, 2008, Uberlândia, Anais... Uberlândia: Universidade Federal de Uberlândia, 2008. 1 CD-Rom.

MATRANGOLO, C.A.R. Dimorfismo sexual em Heilipodus naevulus (Mann.) (Col.: Curculionidae) e impacto do ataque no desenvolvimento inicial de clones de eucalipto. 2010. 141f. Tese (Doutorado em Entomologia) - Curso de Pósgraduação em Entomologia, Universidade Federal de Viçosa, Viçosa, MG.

MORAES, G.J.; BERTI FILHO, E. Coleobrocas que ocorrem em essências florestais. Revista do IPEF, n.9, p.27-42, 1974. Disponível em: <http://www.ipef.br/publicacoes/scientia/nr09/ cap02.pdf>. Acesso em 12 abr. 2012.

SILVA, A.G.A. et al. Quarto catálogo dos insetos que vivem nas plantas do Brasil, seus parasitos e predadores; insetos hospedeiros e inimigos naturais. Rio de Janeiro: Ministério da Agricultura, Laboratório Central de Patologia Vegetal, 1968. 622p.

SORIA, S. de J.; DAL CONTE, A.F. Bioecologia e controle das pragas da videira. Bento Gonçalves: Embrapa Uva e Vinho, 2005. 20p. (Circular Técnica, 63). Disponível em: <www.cnpuv.embrapa.br/publica/circular/cir063.pdf>. Acesso em 12 abr. 2012.

SOUZA, R.M. et al. Condições climáticas associadas com a ocorrência de Costalimaita ferruginea (Fabricius) em plantios de eucalipto. In: SIMPÓSIO DE MEIO AMBIENTE, 5., 2008, Viçosa, MG. Anais... Viçosa: Universidade Federal de Viçosa, 2008. 5p.

SOUZA, R.M. et al. Primeiro registro de Chalcodermus bicolor (Coleoptera: Curculionidae) em plantios de eucalipto. Ciência Rural, v.41, n.3, p.630-633, 2011. Disponível em: <http:// www.scielo.br/pdf/cr/v41n4/a917cr3582.pdf >. Acesso em: 11 abr. 2012. doi: 10.1590/S0103-84782011005000025.

Ciência Rural, v.43, n.2, fev, 2013. 